\title{
A Simulation Study on Demand Disruptions and Limited Resources for Healthcare Provision ${ }^{\star}$
}

\author{
Juliana K. F. Bowles ${ }^{10000-0002-5918-9114]}$, Ricardo M. \\ Czekster2 [0000-0002-6636-4398], Guilherme Alfredo \\ Redeker ${ }^{1[0000-0002-8722-2559]}$, and Thais Webber ${ }^{1[0000-0002-8091-6021]}$ \\ ${ }^{1}$ School of Computer Science, University of St Andrews \\ St Andrews, KY16 9SX, UK \\ $\{j k f b, g r 60, t c w d s\} @ s t-a n d r e w s . a c . u k$ \\ 2 School of Computing, Newcastle University \\ Newcastle upon Tyne, NE1 7RU, UK \\ ricardo.melo-czekster@ncl.ac.uk
}

\begin{abstract}
Philanthropic hospitals in Brazil are in great part funded by the government and are daily accessed by a large portion of the population. As the Brazilian economy faces deep cuts in healthcare, managers are adjusting budgets and focusing on less expensive alternatives such as process improvements. Hospitals are even more impacted by the recent COVID-19 pandemic with widespread disruption on operational processes forcing them to stretch resources. Thus, it brings an opportunity to evaluate the actual performance of these settings under different scenarios where analysts may address bottlenecks and the impact on resources. Our focus is to quantify the capacity of an emergency department to support patient demand with limited resources in pre and postpandemic scenarios. We use a 12-month longitudinal dataset consisting of pre-pandemic emergency occurrences and assigned resources.
\end{abstract}

Keywords: Healthcare processes - Emergency department · Process simulation · COVID-19

\section{Introduction}

Healthcare is a multidimensional domain posing interesting challenges to researchers. Over time, a wealth of studies was carried out on how to combine computing with medical practices while taking into account high quality patient care, balancing budget and available resources 6[12. Despite technological advances and the use of data science or analytics in healthcare sectors across the globe, in some countries those enhancements are far from becoming palpable, as scarce resources and low budget must meet strict objectives mostly within public hospitals 8112. It remains a challenge to redesign complex business processes for performance using what-if scenarios coupled with resource management 36 .

* This research was partially supported by a Scottish Funding Council GCRF grant $2019 / 20$. 
Simulation allows the artificial creation of the most likely scenarios management would face, assigning resources' service time and adjusting capacity to circumvent monetary and physical limitations [15]. These models provide the investigation of key metrics of interest such as average throughput, waiting time, process total capacity, and resource utilisation [10|17, to mention a few. A recent literature review highlighted the importance of the application of Modelling and Simulation (M\&S) specifically to improve operation of emergency wards known as Accidents \& Emergency (A\&E) departments [16. Authors revealed a growing interest on the use of Discrete Event Simulation (DES) as a performance analysis tool in $81 \%$ out of 254 selected papers. Recent studies have applied DES to situations where resource management scenarios exploration was essential to reduce operational costs and increase performance [2]14.

One challenge of tackling Brazilian healthcare is to create a set of comprehensive what-if scenarios to help improve business processes with resourceaware capabilities, especially after the recent Coronavirus pandemic (COVID19) 5[11. According to the World Health Organisation (WHO), Brazil registered 1,085, 038 cases of COVID-19 with 50,617 confirmed deaths by 23 June 2020 18. Even before the global pandemic, A\&E was one of the most crowded departments in Brazilian hospitals. Such developing countries struggle to cope with resource scarcity as in some Brazilian regions difficulties were already a serious predicament prior to COVID-19. It is worth noticing that clinical staff and managers must cope with restrictive resources to meet demand of both patients infected with COVID-19 and those in need of urgent medical assistance.

The focus of this work is to compile information, model, and analyse the ability of an A\&E department to support patient demand with limited resources. We have explored pre-pandemic and post-pandemic simulation scenarios for A\&E settings with increasing daily patient demand, combining available staff (medical doctors and nurses) among different patient arrival rates. Our aim was to investigate the effects of a disrupted demand, for example, that brought forth by COVID-19, to quantify and assess bottlenecks on A\&E operational process and then estimate resource utilisation and queues behaviour. Finally, it is our aim to raise awareness on the possibility of improving resource allocation and patient care in healthcare facilities using M\&S for performance assessment.

This paper is structured as follows. Section 2 covers the issues faced by a Southern Brazilian hospital within the A\&E department while Section 3 presents our M\&S and key measures of interest. Section 4 shows simulation scenarios in pre-pandemic settings with effects on resource allocation and patient waiting time, and numerical analysis of post-pandemic scenarios with demand disruption. Section 5 concludes the work with suggestions and further work.

\section{Southern Brazilian hospital settings}

Our case study is a general public emergency care process on a Southern Brazilian hospital (Hospital Santa Cruz-HSC) considered a reference hospital for cardiac surgeries and traumatology. We focus on the A\&E department as target 
business process for analysis due to high patient demand and equally high amounts of investments needed per year in medical supplies and resources. In 2015, for instance, HSC had performed approximately 221, 500 patient attendances in the A\&E. HSC offers diagnose and treatment of a host of medical procedures such as paediatric and surgery care, among other healthcare services.

The hospital is equipped with a modern medical imaging centre, one inpatient wing, a recovery centre, and an $\mathrm{A} \& \mathrm{E}$ ward operating in two 7-hour and one 10-hour daily shifts: i) 00:00 to 06:59; ii) 07:00 to 13:59; and iii) 14:00 to 23:59. A minimal number of Medical Doctors (MD) and nurses are allocated following a pre-arranged schedule. About the IT (Information Technology) infrastructure, the hospital invested in an Enterprise Resource Planning (ERP) software, which is customised to the hospital needs to maintain data digitised in its business processes [7. A wealth of sub-processes deals with patient arrival and with the outcomes based on clinical decisions. And, to stress things further, Brazilian health regulation mandate a clinical decision in A\&E units to take place within 24 hours.

The hospital underwent serious operational changes to tackle performance, something that is happening since 2015, including IT department remodelling. The effort encompassed all sectors and started from mapping key business processes as well as quantifying available resources. The initiative involved interactive work among stakeholders and managers, staff and performance analysts to discuss improvements in practices and prioritisation. As support tools, they conduct face-to-face meetings, structured interviews, and ERP data analysis.

Furthermore, they have started using the Business Process Model \& Notation $(\mathrm{BPMN})^{3}$ [4 to visually model the business processes with standardised graphical elements and annotations to represent and describe the flow of activities in detail. It is worth mentioning that BPMN offers the possibility of a shared understanding of business processes between administrators and simulation analysts [4, among other advantages.

Recently, the COVID-19 crisis has overwhelmed healthcare organisations across the globe undermining the capacity of operational processes [1118. Thus, it is crucial for hospitals like HSC to prepare for emergent demand disruptions and promote effective decisions to keep patients and staff safe. HSC administrators have reorganised the healthcare services during and post-pandemic especially for most critical processes in A\&E and in the Intensive Care Unit (ICU). The following subsections present the BPMN models elaborated before the emergence of COVID-19 disrupting the A\&E care processes.

\subsection{A\&E Triage System}

The hospital uses the Manchester Triage System (MTS) for clinical risk management as a fundamental system to be applied when the demand exceeds available clinical resources [9]. It helps organising patient flows in $\mathrm{A} \& \mathrm{E}$ wards, considering

\footnotetext{
${ }^{3}$ Object Management Group (OMG) technology standards detailed information on: https://www.omg.org/spec/BPMN/2.0/About-BPMN/
} 
primarily each patients' needs and to ensure high quality care in a timely manner. The triage is performed by a certified trained nurse that assigns a colour to the incoming patient according to a protocol that evaluates the case severity. Fig. 1 presents the BPMN model representing the MTS process.

On patient arrival, a nurse conducts a basic health assessment on the patient, collecting data (temperature, pressure, weight) and a questionnaire on current symptoms. Each case's severity classification is simplified in the BPMN model in two major patient groups: Urgent cases (comprising Red and Orange), and Non-Urgent cases (Yellow, Green, and Blue). The simplification was applied due to few Immediate or Very Urgent care cases retrieved from the ERP software. The bulk is composed of Non-Urgent cases where a significant amount of daily cases cease limited resources in a daily basis.

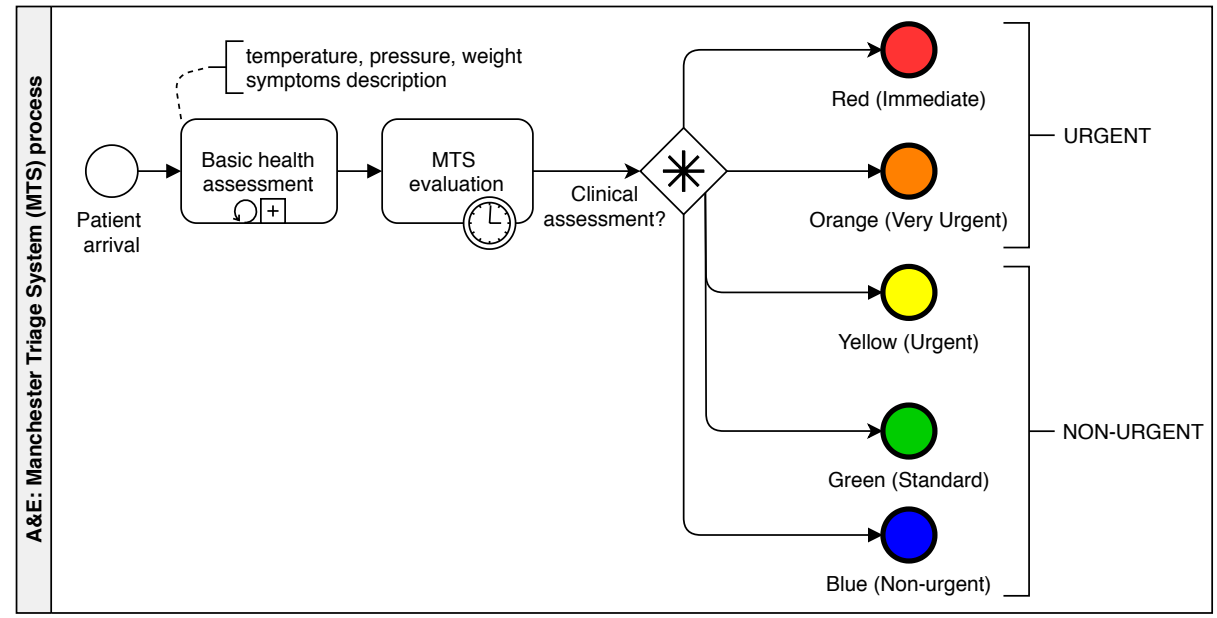

Fig. 1. Manchester Triage System (MTS) process performed by trained nurse.

During the pandemic a pre-triage desk promptly identified COVID-19 patients redirecting them to a new specialised ward whilst the other cases were directed to the usual A\&E. The hospital also moved staff temporarily across departments (e.g., to the ICU, inpatient care ward), keeping only two MDs for A\&E patients not related to COVID-19. The pre-triage on patient arrival only took a few minutes each, which can be considered negligible to the analysis of patient waiting time within the usual operation of the A\&E. During pandemic, the above-mentioned pre-triage contributed to the reduction on patient arrival (i.e., those seeking consultation) in the usual (pre-pandemic) A\&E.

\subsection{Usual pre-pandemic A\&E patient care process}

Patient arrival is a start event in the care process that represents ingress in the system (e.g., by ambulance or as walk-in patient). Depending on severity, the 
colour (Red or Orange) is assigned right before the patient reaches the premises (e.g., immediate or very urgent cases). If it is an immediate case, the patient is automatically classified as Red (urgent case, emergency) and goes straight to a stabilisation sub-process (Fig. 2). After stabilisation (or treatment of an urgent case), one out of three actions happens to patients: i) hospitalisation (ICU or other); ii) Surgery; or iii) discharged with (or without) prescription; also includes 'deceased', i.e., the patient has left the process nevertheless.

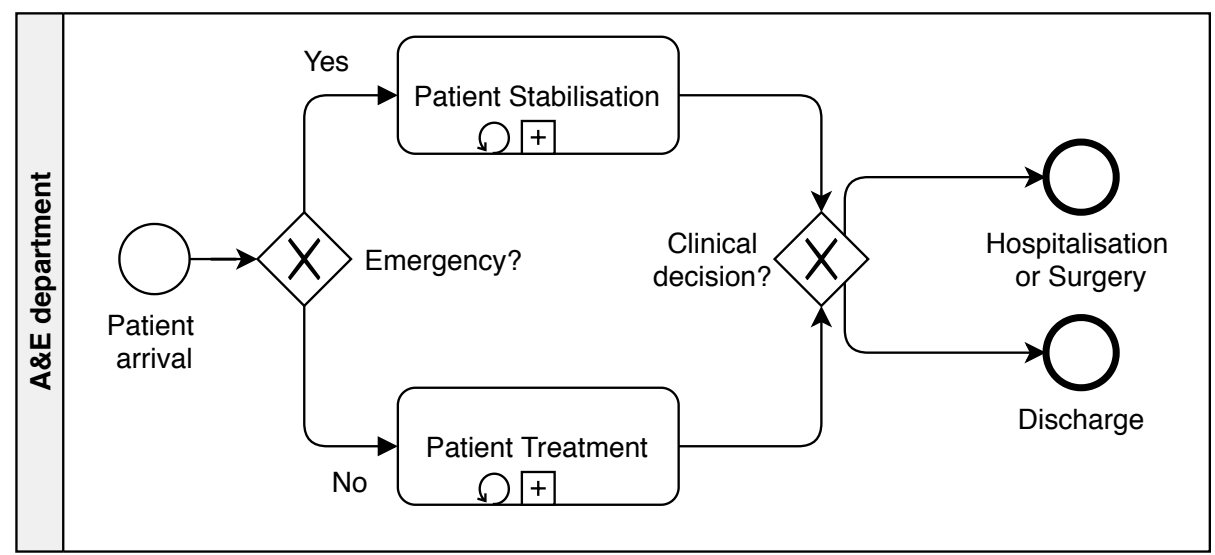

Fig. 2. A\&E macro process.

The urgent cases are contrasted with those non-urgent cases such as patients experiencing minor cuts or burns, mild headaches, or exhibiting amenable cold symptoms, just to name a few. In a non-urgent case, the patient himself goes to Patient Treatment sub-process (Fig. 3).

The patients enter in the activity of Admission registering where the clerk collects more general data (personal data, health details, and other important information such as symptoms and comorbidities). Then, the patient is routed to the Risk assessment activity, where a nurse collects health data using medical devices and a trained nurse performs MTS process.

The patient then goes to a waiting area until an MD is available for consultation (Consult doctor activity). The MD could request more tests (e.g., blood screening, medical imaging, etc.), so the patient goes to Request tests activity; or MD could prescribe medication with immediate administration or just put patient in observation period (Medication \& therapeutics activity) within hospital premises. In both cases, patients should wait for the activities to complete, and sometimes they may return for new consultation with the same MD (or next in the shift) until their treatment process reaches an outcome. 


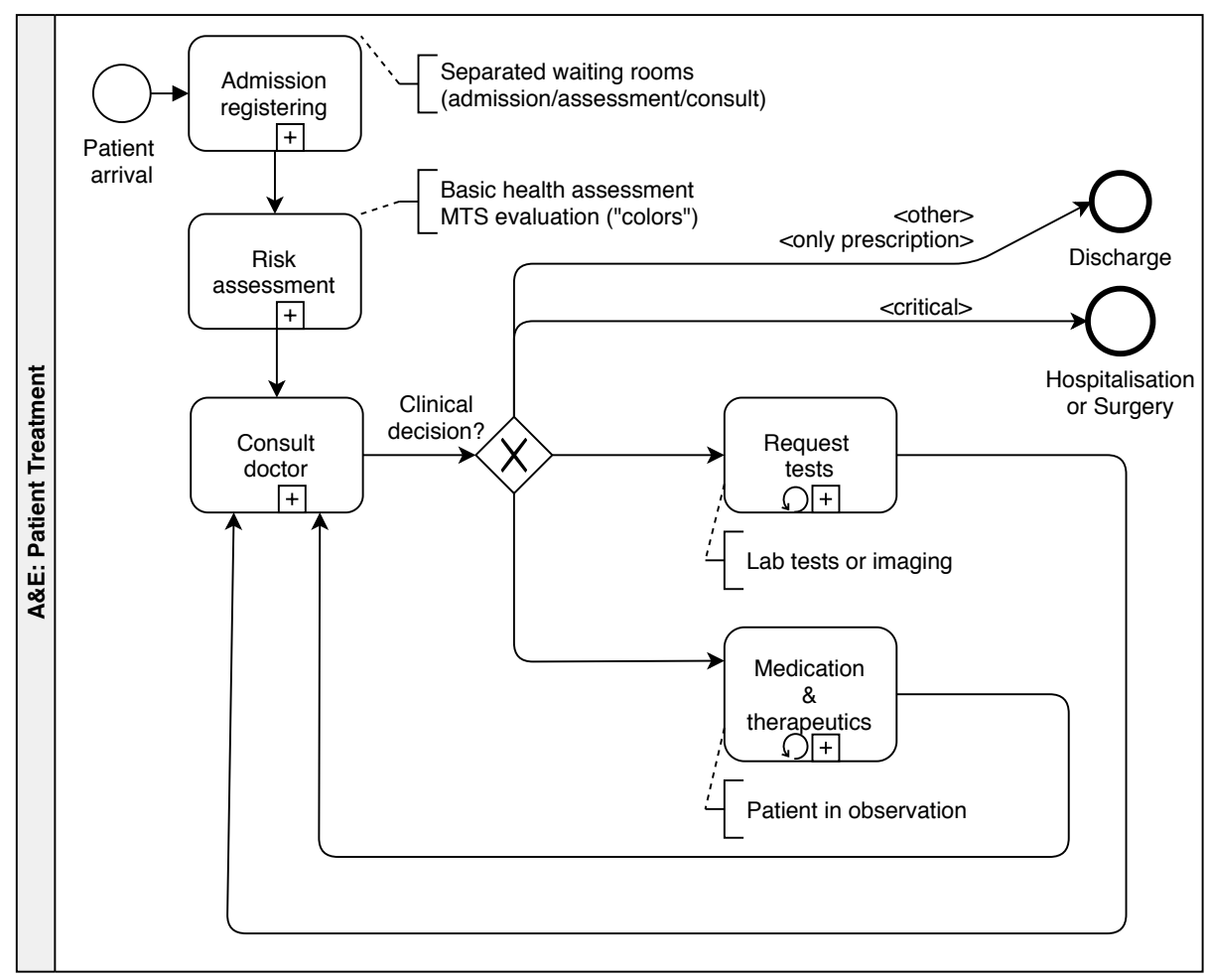

Fig. 3. Treatment sub-process activities until it reaches one of the final outcomes.

\section{Applied Modelling and Simulation in the A\&E}

Modelling and Simulation (M\&S) [17 is a broadly used approach to model systems or processes using primitives that extract quantitative performance indices for analysis. Simulation models based on Discrete Event Simulation (DES) use abstractions and concepts to describe and map processes into descriptive workflows [15]13. The one provided by the BPMN models shown in Section 2 indicates how activities are interconnected and depicts the alternative flows (gateways with decision to be taken according to conditions) present in the process model.

From BPMN models we can manually (or automatically) derive simulation models 411 and execute them in simulation tools based on DES, for instance, the Arena Software ${ }^{4}$ 15. Arena provides an integrated framework for building simulation models in a wide variety of applications from industry to healthcare. It is worth mentioning that the execution of simulation models within any DES framework can help revealing bottlenecks and key resources in almost any process flow model with timing information.

\footnotetext{
${ }^{4}$ Rockwell Arena software information: https://www.arenasimulation.com/
} 
Before the pandemic outset, we have conducted a comprehensive process analysis within the hospital following four basic iterative steps. We aimed to obtain qualitative and quantitative information about processes behaviour and then apply DES technique. Fig. 4 describes the planned steps from initial meetings with stakeholders (Step 1) towards to the actual simulation of the healthcare processes using a simulation tool (Step 4). During development (Step 2 and Step 3 ), the simulation analysts, IT professionals, and stakeholders had interacted to discuss best strategies to deal with ERP data as well as on key modelling aspects.

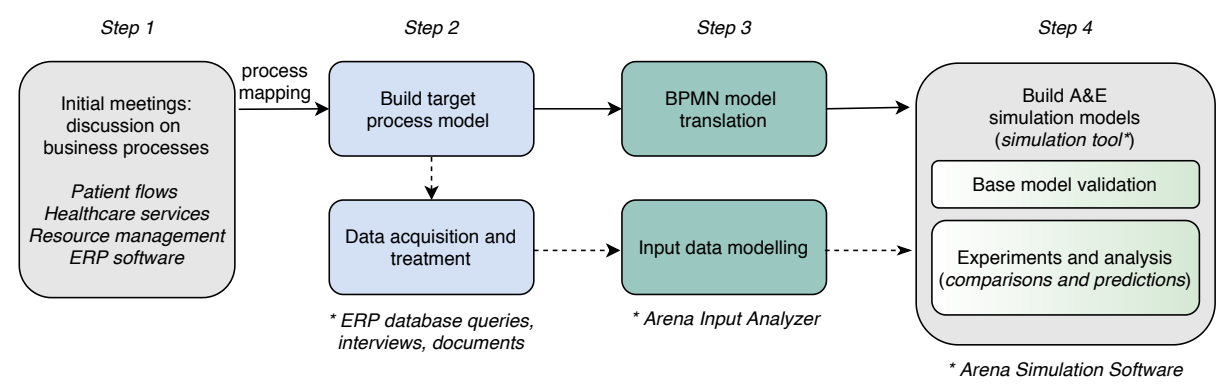

Fig. 4. Planned activities for applying DES in healthcare settings.

The Step 2 produced the BPMN models presented on Section 2 , A comprehensive data acquisition (from documents, textual logs, traces, process monitoring, ERP data) and treatment activity was deemed necessary to: i) detect and filter outliers; ii) process invalid observations due to lack of data inputs by clinical staff; iii) understand how entities and resources are interacting in the process and how the ERP software is processing the entries, and; iv) discover how the actual process operates. In the event of data inconsistencies (e.g., invalid or missing numbers, outliers), analysts should use appropriated statistical techniques to overcome the issues [15]. Arena software also provides a statistical tool named Arena Input Analyser [15] that is a built-in tool to provide basic statistical properties (e.g., minimum, maximum, mean, standard deviation, and so on) as well as probability distribution fitting to datasets, which is conducted on Step 3 and applied on Step 4.

On Step 3, BPMN models are ready to be translated to simulation models. The flow is directly mapped to DES in terms of structure because both have similar elements (activities, start/end events, and decision gateways). After data acquisition on Step 2, the input data modelling activity provides a probability distribution function to parametrise the simulation models (patient arrival rate, activities service times, resources' capacity, and rotation probabilities on decision gateways). Thus, model parametrisation depends on acquiring sets of input data from reliable sources, including database sets and personnel interviews to review inconsistencies. In our case study, we have chosen to model the decision gateways with constant probabilities for output flows. For example, it was verified in the 
dataset that around $50 \%$ of patients' clinical path have followed an outcome of 'Discharge'. Thus, the majority of A\&E patients were discharged without needing further tests, or medication applied on site.

The Step 4 is the refinement of the simulation model (DES) translated from the BPMN model, adding timing information such as the probability distribution expressions, also resources quantity and behaviour, i.e., adding the parameters obtained on Step 3 input data modelling. Our previous research 4 has proposed BPMN models embedded with a structured format for text annotations to improve simulation design and what-if scenario management. At the Step 4 analysts can make use of several statistical tools and validation approaches they see fit to their experiments. In the end, a baseline model is evaluated towards the measures of interest (e.g., average resource utilisation, queuing analysis such as waiting time and queue size, and total process capacity, to mention a few). For example, the patient waiting time could be calculated from the acquired dataset or discussed with stakeholders, and then its difference from the figure yielded by the simulation model should not be statistically significant.

After model validation with stakeholders, analysts start planning the scenarios on patient arrival rates, resource allocation, service times, and decision gateways' probabilities. During scenarios execution, analysts observe the process behaviour and statistical calculations are summarised in performance indices [17. The analysts can then evaluate these indices for multiple what-if scenarios. Performance scenarios enable stakeholders to interpret and act on changes or suggest modifications that would probably be less costly, or use fewer resources for the same or higher desired output. M\&S offers a way to diminish the impact of physical changes and show managers how simple adjustments could yield better outcomes [15]. Next section details the Input data modelling (Step 3), and the simulation scenarios and analysis (Step 4).

\subsection{Input data modelling for the pre-pandemic simulation}

During the data acquisition activity (Step 2) across hospital IT infrastructure, we inspected 221,664 daily operation logs to use as real parameters for the baseline model. We have pointed out the following issues in the dataset:

1. Seasonality aspects: we have analysed the one-year dataset to determine whether or not the seasons (or the day of the week) were affecting our study, accumulated monthly;

2. Arrival patterns: we investigated the rates of arrival (10,185 entries), according to severity, and per hour of the day, accumulated monthly;

3. Consultation times for medical staff: we were interested into determining the average time spent per medical staff on patients care according to severity.

Fig. 5 presents seasonality aspects by month for each aggregated group - Urgent and Non-urgent (refer to Fig. 1). It shows the percentage on the total number of occurrences observed throughout the duration of the study (12 months), matching the occurrences described in the BPMN model (refer to Fig. 3). 


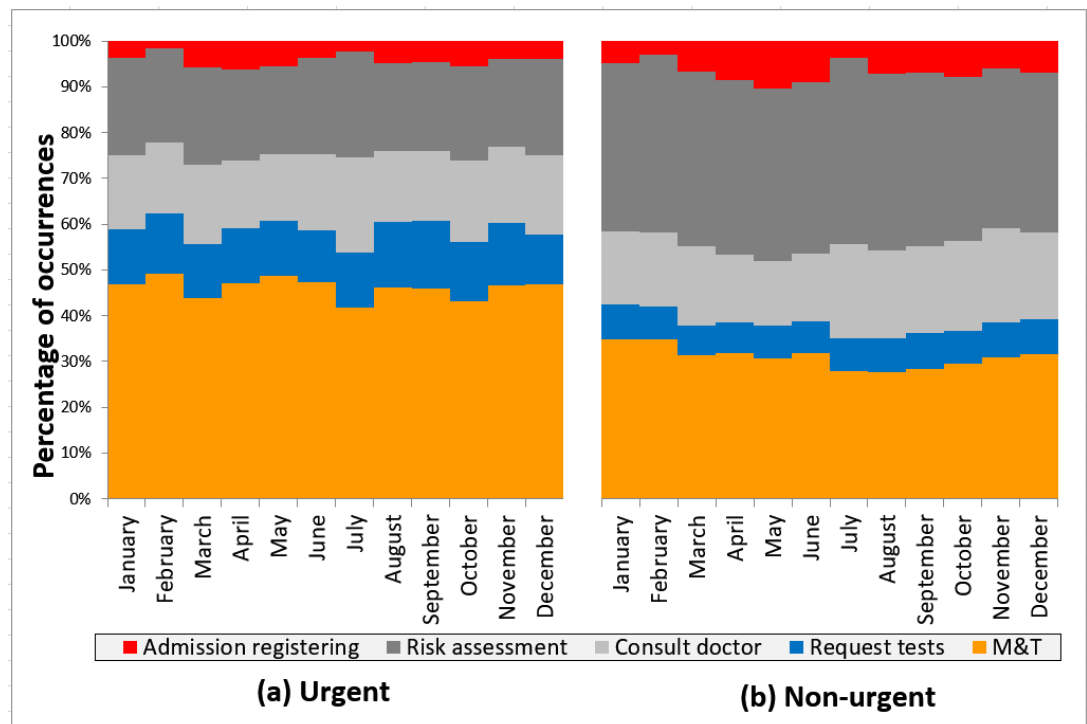

Fig. 5. Seasonality (by month) for Urgent (a) and Non-urgent (b).
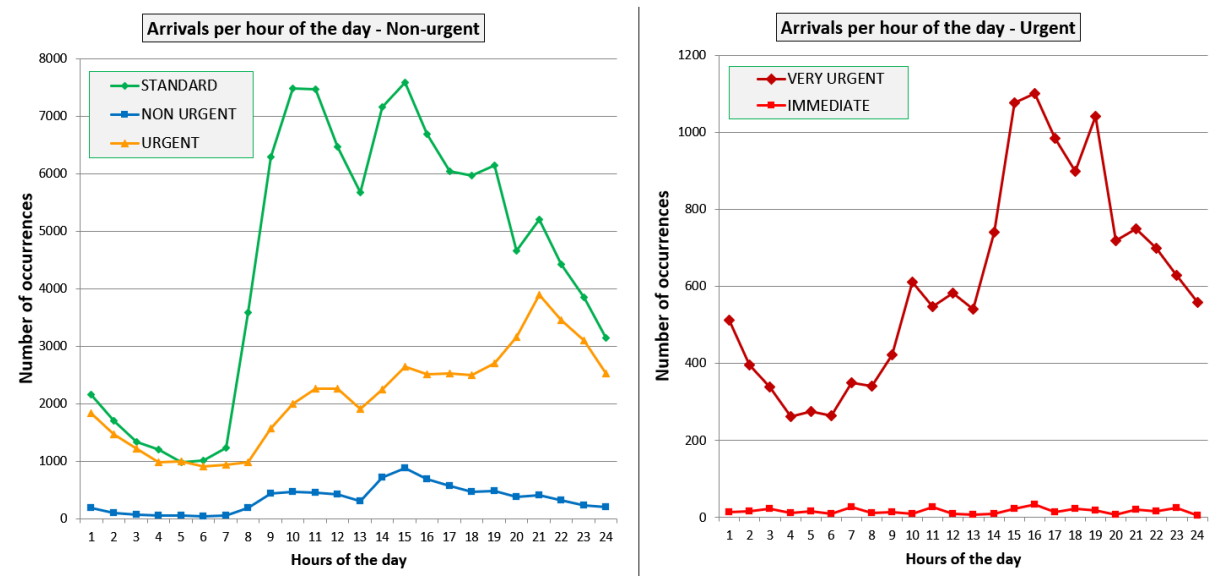

Fig. 6. Arrivals per hour (accumulated monthly) in Urgent (a) and Non-urgent (b). 
Regarding to patient arrival patterns (per hour of the day), Fig. 6 shows the number of arrivals for Urgent and Non-urgent cases. It presents a low number of occurrences prior to the beginning of the day (i.e., before 6:00), which increases as the day passes, and decreases towards the end. Fig. 6(a) shows an interesting behaviour after 12:00 where the incidence increases considerably when taking into account the totality of occurrences. However, because those cases concern Urgent cases, the total number is considered very low (around 300 occurrences), so no actual interpretation is effective for these cases.

Table 1 relates the inter-arrival time and the human resources to known probability distributions. We used the Arena Input Analyser tool that operates over a list of data points acquired from the data. Probability distributions were generated for each activity in the simulation flow. We show the descriptive statistics from the dataset before data treatment. It indicates the present of outliers, which supposedly incurred from ERP software misuse (most probably by human error while inputting data) or business process related particular issues, concerning their daily operation. We computed the probability distribution expressions after data treatment step and used the values to parametrise the simulation models.

We direct attention to the variability of MDs' service time for both Consultation and M\&T activities, since the amplitude is high, e.g. from 0.2 to 2, 400 minutes and from 1 to 1,220 minutes, respectively.

Table 1. Statistical analysis in the input data modelling step.

\begin{tabular}{|c|c|c|c|c|c|c|}
\hline $\begin{array}{c}\text { Resource } \\
\text { (Task) }\end{array}$ & $\begin{array}{c}\text { Data } \\
\text { points }\end{array}$ & Min. & Max. & Mean & $\begin{array}{c}\text { Std. } \\
\text { Dev. }\end{array}$ & $\begin{array}{c}\text { Probability distribution } \\
\text { (scale in minutes) }\end{array}$ \\
\hline \hline $\begin{array}{c}\text { Patient } \\
\text { inter-arrival }\end{array}$ & 10,185 & 0 & 119 & 15.5 & 19.2 & $-0.001+$ EXPO(15.5) \\
\hline $\begin{array}{c}\text { Desk clerk } \\
\text { (Admission Reg.) }\end{array}$ & 66,702 & 0 & 44 & 0.713 & 1.02 & $-0.5+$ GAMM $(0.563,2.16)$ \\
\hline $\begin{array}{c}\text { Qualified Nurse } \\
\text { (Risk Assessment) }\end{array}$ & 67,297 & 0 & 35 & 0.513 & 0.903 & $-0.5+\operatorname{EXPO}(1.01)$ \\
\hline $\begin{array}{c}\text { Nurse } \\
\text { (Request Tests) }\end{array}$ & 843 & 0 & 202 & 2.56 & 10.3 & $-0.001+$ EXPO (2.56) \\
\hline $\begin{array}{c}\text { Medical Doctor } \\
\text { (Consultation) }\end{array}$ & 33,174 & 0.2 & 2,400 & 50 & 159 & WEIB (13.1, 0.505) \\
\hline $\begin{array}{c}\text { Medical Doctor } \\
\text { (M\&T) }\end{array}$ & 43,463 & 1 & 1,220 & 4.2 & 15 & $0.999+$ WEIB (0.216, 0.259) \\
\hline
\end{tabular}

We have performed a time analysis for the top five MDs by time intervals (of 5 minutes), dividing consultation duration in nine categories ranging from $0-4$ minutes to 40-44 minutes. The most usual consultation duration is of 0-4 minutes, followed by 5-9 minutes, and then 10-14 minutes. Beyond 15 minutes very few occurrences are present for Non-urgent cases. We discovered that doctors and staff use the information system only to close or report occurrences, allowing 
patients to move along the process as quick as possible. After interviews, MDs described their actual consultation period with the patient around 15 minutes on average. The remaining categories had low frequency, i.e., from 45-49 minutes or more, could be considered as outliers in the dataset according to administrators (i.e., adverse events occurring during consultation, or even a particular operation of the ERP software).

As mentioned earlier, using data points from ERP data is crucial determining baseline parameters and asserting model validity when performing experiments. Preliminary simulation experiments set the statistical parameters and analysts can check whether the baseline model is simulating the current process operation or need further improvement. Fig. 7 illustrates the structured annotations documented in the BPMN model to guide the simulations construction.

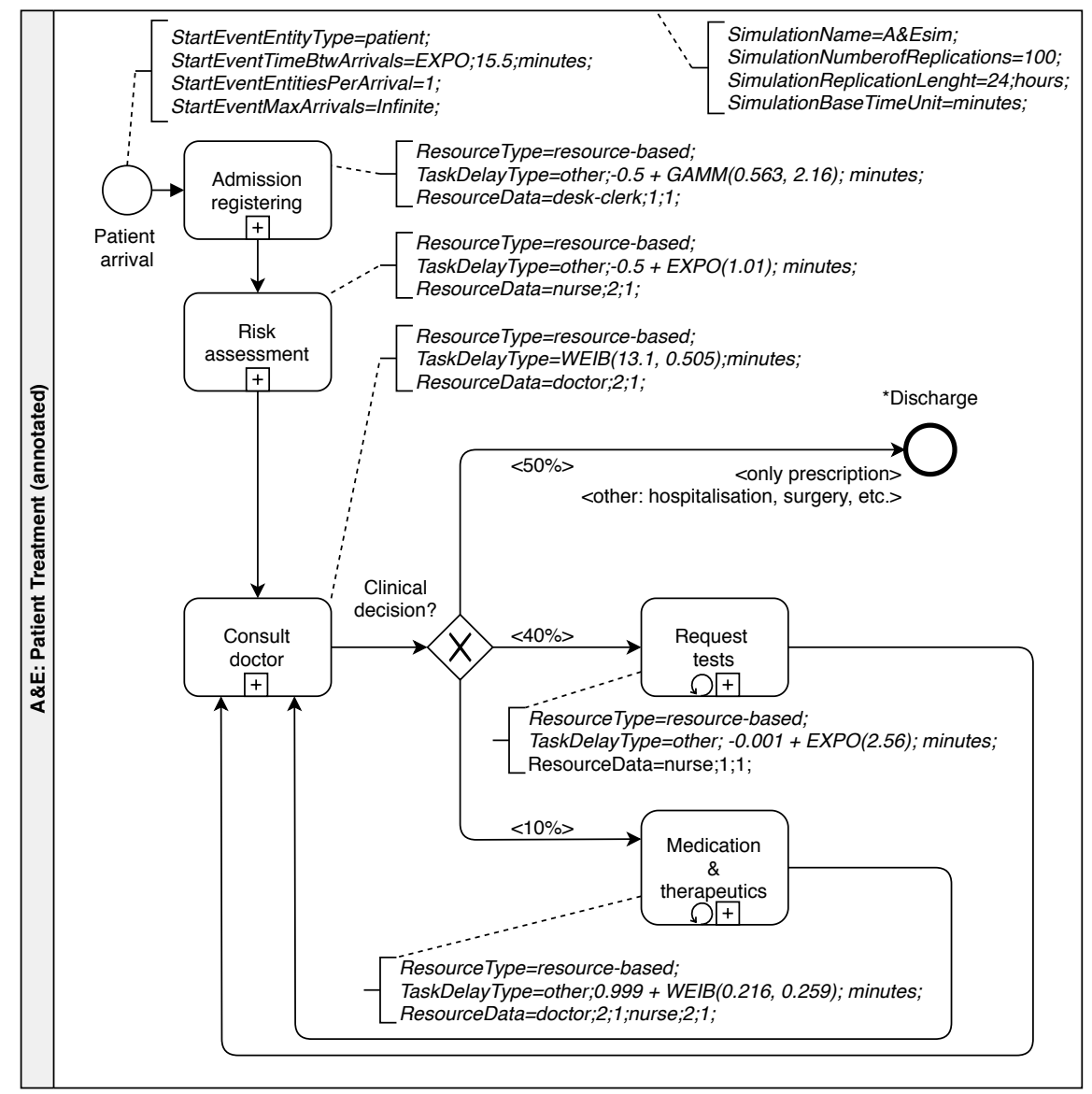

Fig. 7. An example of annotated BPMN model for simulation purposes. 


\section{Pre-pandemic and post-pandemic scenarios simulation}

The pre-pandemic scenario modelled the busiest hour for key human resources in the A\&E, i.e., selecting the parameters for the worst case present in the real-case hospital before COVID-19 outset, then aligning the next what-if scenarios accordingly. We considered three simulation scenarios for arrival rates (pre-pandemic, pandemic and post-pandemic) and compiled the results in Fig. 8 Fig. 11. We have assumed inter-arrival time of incoming patients to be constant throughout the workday, as well as service time. The values come from input data modelling step (Fig. 7). As output, we were interested in performance indices such as patient waiting time, number of patients waiting for service, and resource (MDs, nurses) utilisation. The Clerk utilisation in the A\&E process is not considered as an issue by hospital managers after management installed a service totem. It has optimised patient check-in and organised triage screening. Staff interviews pointed out that the hospital's major concern was related to consultation waiting room and on urgent care services provided to the patient.

\subsection{Pre-pandemic scenario simulation}

The pre-pandemic scenario (baseline scenario) uses an exponentially distributed inter-arrival time based on the average time between occurrences yielded as 15.5 minutes by the statistical fitting tool in Fig. 1. The most requested resource in the whole process is the MD as confirmed by earlier interviews (Step 1 and 2), and thus it is replicated in the simulation behaviour (Step 4) according to the MD utilisation plot in Fig. 8(a). The two available MDs in the Patient Treatment sub-process operate in a shared-based routine on their activities (Consult doctor and Medication \& therapeutics - M\&T), both being dedicated $100 \%$ of the time in their A\&E shift catering patients. Thus, if an MD is not consulting a patient at a given time, then she/he could be working in the M\&T activity, and vice-versa.

In addition, two nurses work on their respective activities (Risk Assessment and Request tests) also in a shared-based mechanism. Results on Nurse utilisation in Fig. 8(d) show that the A\&E nurses could stay idle in pre-pandemic settings since they mostly execute Risk assessment activities during their shifts, and only perform M\&T depending on consultation outcome, thus they are shared only if there is demand (patient arrival). The probability of M\&T is less than $10 \%$ according to the dataset. The majority of patients seeking out consultations (around 90\%) are discharged with prescriptions or requested tests, without the need of monitoring or medication administration in situ.

During interviews, managers and staff mentioned other sub activities performed by nurses and doctors (e.g., filling forms, preparing medications, cleaning/preparing rooms) during their shifts. These sub activities are not explicitly described in the BPMN mapping of the A\&E Patient Treatment process, so the resource utilisation indice refers only to the performance on modelled activities. We have validated our simulation approach matching the baseline model result (pre-pandemic scenario) with the dataset statistical analysis, focusing on the average number of patients waiting during peak hours, i.e., the average queue size 

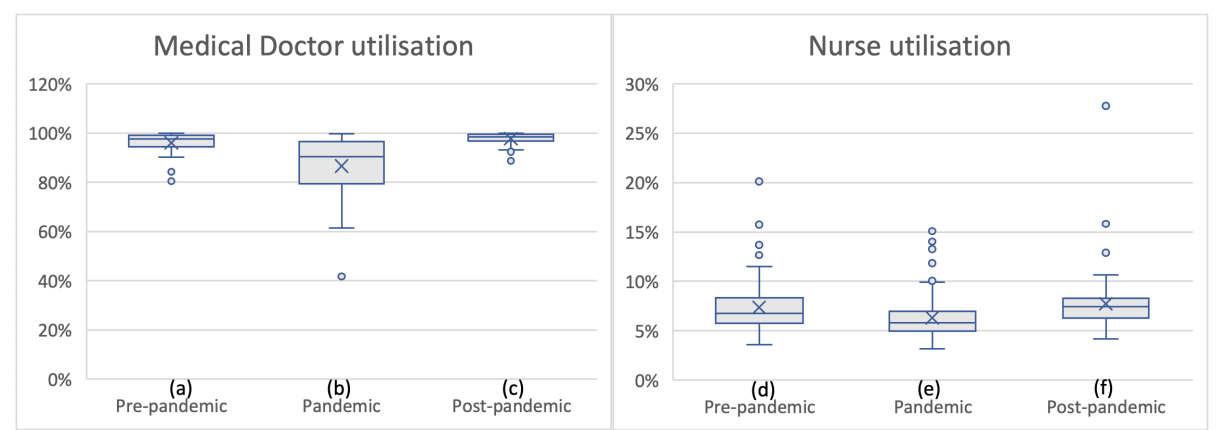

Fig. 8. Resource utilisation simulation results.
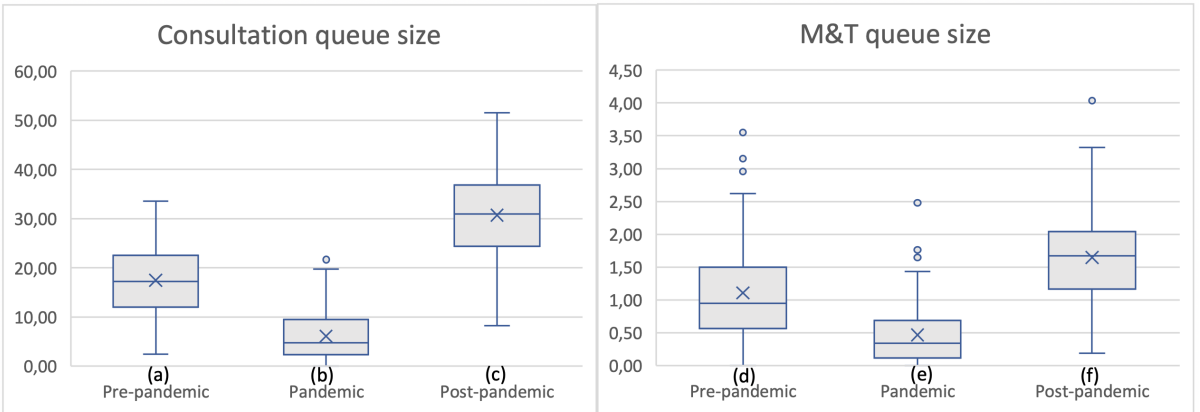

Fig. 9. Patient queue simulation results.

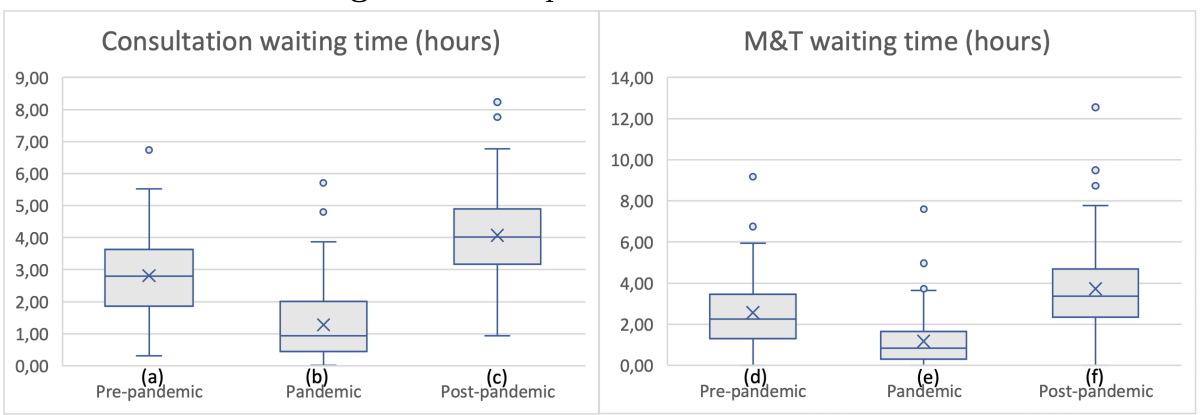

Fig. 10. Patient waiting time simulation results.

(in peak operation hours) within $95 \%$ confidence interval. The patient waiting time is the key performance indice we have collected for the three distinct scenarios under evaluation. Results in Fig. 9(a) demonstrate an average number of 17.48 patients in queue waiting consultation and in Fig. 10(a) the average patient waiting time around 2.8 hours in the pre-pandemic setting. Moreover, Fig. 9(d) and Fig. 10(d) provide the simulation results on M\&T. Comparatively, patients undergoing treatment after consultation also presented an elevated waiting time 
as shown in consultation outcomes, i.e., the waiting time was around 2.56 hours despite an average number of 1.10 patients waiting in queue. This simulation provided that the total patient waiting time is 4.33 hours (in average), confirmed by the managers for their worst-case scenario of observed waiting time.

For improving waiting time and queue size for the pre-pandemic scenario, we suggested the addition of another MD. The new scenario speeds up the Consultation queue in the $\mathrm{A} \& \mathrm{E}$, where the total patient waiting time was reduced from 4.33 to 2.06 hours (in average) and the queue from 17.48 to 7.83 patients waiting consultation. The performance bottleneck still persists in the waiting time on Consultation activity, however, for the managers perspective, has increased patient overall satisfaction.

During interviews, managers mentioned that their knowledge about bottlenecks in A\&E operation was acquired mostly by daily in situ observations and casual patient satisfaction enquiries made in waiting rooms often by temporary staff. They highlighted that the waiting rooms capacity were struggling to accommodate the pre-pandemic demand (mostly in peak days) and the total waiting time was rightly inferred by the baseline simulation. Although managers discussed that the baseline model lacks of adverse events that could worsen the patient waiting time, for instance, when systems become unavailable (e.g., reception totem malfunction) or healthcare services are overloaded (e.g., delays in exam results delivery).

\subsection{A\&E disruption scenarios during and post-pandemic}

The COVID-19 pandemic brought new challenges and policies for hospitals and emergency care regarding their services provision. In the A\&E for instance, activities in situ such as consultations were dramatically reduced. Managers reported a $50 \%$ reduction on face-to-face assistance to patients, and scheduled resources (MDs and nurses) started performing new activities concerning the urgent actions needed by the pandemic (e.g extra time to put protective equipment or cleansing). An isolated service ward separated from A\&E was set to treat only COVID-19 infections. MDs in the A\&E received new shared tasks, however their priority were to consult patients arriving at the emergency ward as expected in the pre-pandemic scenario.

Our second simulation scenario is a $50 \%$ reduction on inter-arrival time in $\mathrm{A} \& \mathrm{E}$ (exponent. distr. as 23.25 minutes). Fig. 8 (b) shows a high variability on MDs' utilisation, so the reduction on patients arrival provided room for MDs is shared in new activities. In Fig. 8(e) Nurses reduced utilisation, i.e. they could operate in a reduced number in $\mathrm{A} \& \mathrm{E}$ or having shared responsibilities on new activities. Waiting time was reduced from 2.80 to 1.28 hours on average in Consultation activity during pandemic as observed in Fig. 10(b), and queue size from 17.48 to 6.12 patients on Fig. 9(b). Regarding M\&T in Fig. 10(e) and Fig. 9(e), waiting time was reduced to 1.17 hours representing a $50 \%$ drop in services provided with less than one queued patient during workdays. Both reductions were caused by the decrease in urgent care (less people on the streets, fewer car/work related accidents) and by the patients' concern on being contaminated when 
accessing the hospital premises (avoiding visits). The simulation results showed that MDs and nurses (A\&E capacity) were sufficient to assist non-urgent cases which are not related to COVID-19 during the pandemic.

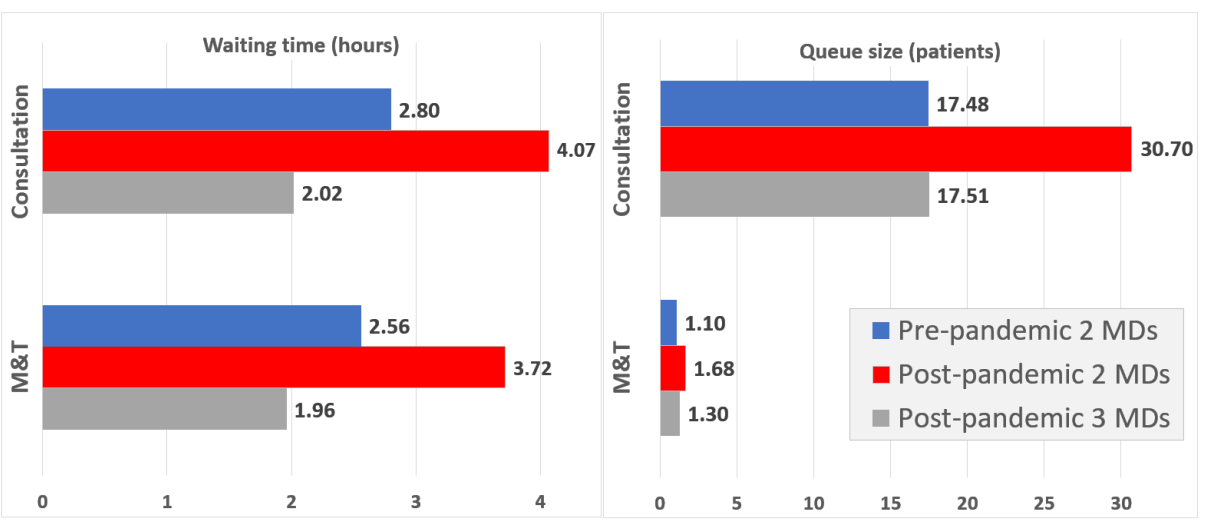

Fig. 11. Comparison of pre-pandemic and post-pandemic scenarios.

A post-pandemic effect on hospital operations is predicted by managers and MDs with bottleneck even more evident especially on $A \& E$ and other services interrupted such as cancer treatments, surgeries scheduling, and several others. The arrival rates were suppressed during pandemic period and when services return to normal operation more patients will seek care for their health issues in minor and major cases. We have increased the inter-arrival patient time by $25 \%$ in the post-pandemic scenario in comparison with pre-pandemic, assigning an exponentially distributed value of 11.63 minutes. This increase represents mostly non-urgent patients (e.g., patients with minor to moderate injuries, mild symptoms, or those in the identified risk group for COVID-19) that avoided the hospital premises during the pandemic. Fig. 8(c) shows MDs' utilisation will be close to $100 \%$ all the time, meaning that after post-pandemic wave slows down, resources in the $\mathrm{A} \& \mathrm{E}$ are to be increased to reduce their utilisation rate to levels similar to pre-pandemic settings. Nurses suffered an increase on utilisation rate but still less than $8 \%$ (in average).

Patient waiting time for consultation has doubled in post-pandemic scenario with two MDs, from 2.80 hours to 4.07 hours as shown in Fig. 11. The queue size has increased from 17.48 to 30.70 patients similarly, which represents an even worst scenario to be faced regarding patient experience in the waiting rooms. For that reason, we have simulated an alternative post-pandemic scenario increasing capacity to three MDs and the simulation results presented in Fig. 11. It showed an improvement in waiting time (about 2 hours for consultation) that could be compared to pre-pandemic scenario with two MDs (2.80 hours), and also queue size was computed as 17.51 patients, in average. 


\section{Conclusion}

M\&S is crucial to accurately model diverse situations in healthcare. When models are parametrised with actual data, they provide valuable insights to managers as to how to best allocate (limited) resources or cope with increasing demand, to mention a few advantages. This is especially relevant with major unanticipated events such as what the world is now witnessing with COVID-19. Emerging countries are particularly interested in such results as the situation has only worsened after the pandemic. The problem will persist even after the pandemic subsides, as there as no vaccines for the whole population and, on top of that, it is unfeasible to think about a $100 \%$ coverage that could exterminate the virus throughout the globe. It is thus only reasonable to prepare for a constant increase in these respiratory issues cases with higher contagion levels.

This work highlighted the importance of M\&S on actual settings and the need for better collecting input data for more consistent quantitative measures. It seems like this is a solved problem, however, in emerging countries buying less expensive ERPs, this is very challenging and a source of concern. Hospital nurses and MDs were trained to input better data into the ERP software and interact with the modelling sector to improve the process models even further.

As limitations of our work we may cite that it could fall short in terms of adaptability to new situations, i.e., it has a fixed evaluation quality that it is hard to circumvent whenever a crisis or an anticipated behaviour is bound to occur. In this sense, we hope that the models could be quickly revised and re-executed, where specialised personnel would pinpoint shortcomings on the process and advise the medical staff in a timely manner.

Our work has considered pre and post-pandemic scenario analysis varying inter-arrival time and resource allocation to specific tasks in $\mathrm{A} \& \mathrm{E}$ of a sizeable hospital in Brazil. The results have discussed the impact of the pandemic on the hospital A\&E department, helping managers devise new operational strategies to cope with demand and address better allocations.

As future work, we aim to expand the model with other activities and assign resources to tasks to increase the modelling opportunities of more realistic settings. We also would like to review service times and suggest improvements to management as to deadlines so MDs and nurses could meet to increase operational efficiency due to adversities, and deal better with process bottlenecks.

\section{Acknowledgements}

We thank HSC managers and staff for the research opportunity and the time spent on interviews as well as sharing datasets from their healthcare system. We are grateful to Dr Sandra Quickert and Dr Marco B. Caminati for discussions on input data analysis.

\section{References}

1. Antonacci, G., Calabrese, A., D'Ambrogio, A., Giglio, A., Intrigila, B., Ghiron, N.L.: A BPMN-based automated approach for the analysis of healthcare processes. 
In: Proc. of the 25th Int. Conf. on Enabling Technologies: Infrastructure for Collaborative Enterprises (WETICE). pp. 124-129. IEEE Computer Society (2016)

2. Baril, C., Gascon, V., Vadeboncoeur, D.: Discrete-event simulation and design of experiments to study ambulatory patient waiting time in an emergency department. Journal of the Operational Research Society 70(12), 2019-2038 (2019)

3. Bisogno, S., Calabrese, A., Gastaldi, M., Ghiron, N.L.: Combining modelling and simulation approaches: How to measure performance of business processes. Business Process Management Journal 22, 56-74 (2016)

4. Bowles, J., Czekster, R.M., Webber, T.: Annotated BPMN models for optimised healthcare resource planning. In: Software Technologies: Applications and Foundations - STAF 2018 Workshops. Springer LNCS, vol. 11176, pp. 146-162 (2018)

5. Carenzo, L., Costantini, E., Greco, M., Barra, F.L., Rendiniello, V., Mainetti, M., Bui, R., Zanella, A., Grasselli, G., Lagioia, M., Protti, A., Cecconi, M.: Hospital surge capacity in a tertiary emergency referral centre during the covid-19 outbreak in italy. Anaesthesia 75(7), 928-934

6. Costa, L.B.M., Filho, M.G., Rentes, A.F., Bertani, T.M., Mardegan, R.: Lean healthcare in developing countries: evidence from Brazilian hospitals. Int. J. of Health Planning and Management (2017)

7. Czekster, R.M., Webber, T., Jandrey, A.H., Marcon, C.A.M.: Selection of enterprise resource planning software using analytic hierarchy process. Enterp. Inf. Syst. 13(6), 895-915 (2019)

8. Doniec, K., Dall'Alba, R., King, L.: Brazil's health catastrophe in the making. The Lancet (July 2018)

9. FitzGerald, G., Jelinek, G.A., Scott, D., Gerdtz, M.F.: Emergency department triage revisited. Emergency Medicine Journal 27(2), 86-92 (2010)

10. Günal, M., Pidd, M.: Discrete event simulation for performance modelling in health care: a review of the literature. Journal of Simulation 4, 42-51 (2010)

11. Heymann, D.L., Shindo, N.: COVID-19: what is next for public health? In: The Lancet, vol. 395(10224), pp. 542-545 (Feb 2020)

12. Hussain, M., Malik, M.: Prioritizing lean management practices in public and private hospitals. J. of Health Organization and Management 30(3), 457-474 (2016)

13. Law, A., Kelton, W.: Simulation modeling and analysis - third edition. McGraw-Hill, 3 edn. (2000)

14. Pongjetanapong, K., Walker, C., O’Sullivan, M., Lovell-Smith, M., Furian, N.: Exploring trade-offs between staffing levels and turnaround time in a pathology laboratory using discrete event simulation. The Int. J. of Health Planning and Management 34(2), e1119-e1134 (2019)

15. Rossetti, M.D.: Simulation Modeling and Arena. Wiley Press, 2 edn. (2010)

16. Salmon, A., Rachuba, S., Briscoe, S., Pitt, M.: A structured literature review of simulation modelling applied to emergency departments: Current patterns and emerging trends. Operations Research for Health Care 19, 1-13 (2018)

17. Sokolowski, J.A., Banks, C.M.: Principles of modeling and simulation: a multidisciplinary approach. John Wiley \& Sons (2011)

18. WHO: World health organisation, covid-19 dashboard, country: Brazil (2020), https://covid19. who.int/region/amro/country/br, accessed on June 23, 2020 